\title{
Hepatocellular Injuries Observed in Patients with an Eating Disorder Prior to Nutritional Treatment
}

\author{
Masayo Tsukamoto ${ }^{1}$, Atsushi Tanaka ${ }^{2}$, Motoe Arai ${ }^{1}$, Naoki Ishii ${ }^{1}$, \\ Daisuke Ohta ${ }^{3}$, Noriyuki Horiki ${ }^{1}$ and Yoshiyuki Fujita ${ }^{1}$
}

\begin{abstract}
Background/Aims Although hepatocellular injuries are occasionally observed in patients with an eating disorder, such as anorexia nervosa (AN), it remains unclear how malnutrition in patients with AN causes hepatocellular damage. In this retrospective study, we aimed to reveal the characteristics of hepatocellular injuries in patients with an eating disorder without any nutritional treatment, to eliminate the possible hepatotoxic effects of nutritional support.

Subjects and Methods Twenty-five patients with an eating disorder who visited St. Luke's International Hospital were enrolled. No nutritional treatment had been performed for these patients. The diagnosis of eating disorder as well as typing (anorexia nervosa or bulimia nervosa) was made according to the Diagnostic and Statistical Manual of Mental Disorders (DSM-4). We reviewed the charts of these patients and examined the clinical parameters.

Results Elevation of serum alanine aminotransferase (ALT) was found in 13 out of 25 (52\%), all of whom were categorized as AN. In 13 AN patients with elevated ALT, the median of serum ALT values was 92 [39438] IU/L. The body mass index (BMI) was significantly lower (13.5 vs. 17.3, p=0.011), and the duration since the onset of AN was shorter (4.2 years vs. 8.9 years, $\mathrm{p}=0.037$ ) in patients with elevated ALT. The age was younger in patients with elevated ALT, even though not significant ( 24.5 vs. 29.8 , $p=0.139$ ). Logistic regression analysis revealed that only BMI was a significant determinant for the development of hepatocellular injuries $(\mathrm{OR}=3.46$; $95 \%$ CI 1.06-11.34, $\mathrm{p}=0.041)$. Imaging studies failed to demonstrate any abnormalities, including fatty liver.
\end{abstract}

Conclusion The current study indicated that lower BMI might significantly contribute to the development of hepatocellular injuries in AN patients prior to any nutritional treatments.

Key words: fatty liver, anorexia nervosa, bulimia nervosa

(Inter Med 47: 1447-1450, 2008)

(DOI: 10.2169/internalmedicine.47.0824)

\section{Introduction}

The eating disorder is characterized by abnormal eating behaviors with a trouble of making body image and a strong desire to be slim. According to DSM-4 (Diagnostic and Statistical Manual of Mental Disorders), the eating disorder is categorized as anorexia nervosa (AN) and bulimia nervosa (BN) (1). In general, patients with AN refuse to maintain body weight within a normal range, with a fear of weight gain and profound disturbance of body image. By contrast, patients with $\mathrm{BN}$ usually experience the episodes of binge eating without appropriate controls, followed by compensatory behavior of the purging type (self-induced vomiting) or non-purging type (excessive exercise) (1)-.

It has been reported that more than $10 \%$ of patients with AN demonstrated elevation of aminotransferases $(2,3)$. While hepatocellular injuries are usually mild and spontaneously improved to normal range along with the improvement of the nutritional state (2), there are several case re-

${ }^{1}$ Department of Internal Medicine, St. Luke's International Hospital, Tokyo, ${ }^{2}$ Department of Medicine, Teikyo University School of Medicine, Tokyo and ${ }^{3}$ Department of Psychosomatic Medicine, St. Luke's International Hospital, Tokyo

Received for publication December 13, 2007; Accepted for publication May 16, 2008

Correspondence to Dr. Masayo Tsukamoto, tukamsa@luke.or.jp 
ports describing severe hepatic failure due to eating disorders, occasionally leading to fatal outcome (4-7). The mechanisms and pathogenesis of hepatocellular injuries in patients with eating disorder still remain unclear. Whether excess accumulation of fat in the liver contributes to the elevation of liver enzymes in eating disorder is yet controversial (2, 7-10). Although it is clear that nutritional deficiency plays a crucial role in establishing liver injuries in eating disorders, the clinical manifestations are not alike to those observed in well-known malnutrition state, such as Kwashiorkor, and are rather very diverse depending on patients, and clinical course even in a single patient (11). This heterogeneity of clinical features would be partly explained by the fact that hepatocellular injuries might be brought about by not only eating disorders itself but modification with nutritional treatment, including total parental nutrition (TPN) (12, 13).

Therefore, in this retrospective study, we aimed to reveal the characteristics of hepatocellular injuries in patients with eating disorder without any nutritional treatment, by the enrollment of untreated patients in whom the diagnosis of eating disorders were just established.

\section{Materials and Methods}

\section{Patients}

Twenty-five patients, who visited the Department of Psychosomatic Medicine, St. Luke's International Hospital during the period from January 2004 to July 2007 and were diagnosed as having eating disorders without any previous nutritional treatment. We enrolled these 25 patients with eating disorders in the current retrospective study. The diagnosis of eating disorder was made based on the history taking from patients and family members, and according to the DSM-4, proposed by the American Psychiatric Association (1). We reviewed the charts of these patients and examined clinical parameters possibly associated with hepatocellular injuries, such as age, sex, height, weight, type of eating disorders ( $\mathrm{AN}$ or $\mathrm{BN}$ ), duration since the onset of the disease. The results of blood tests, including complete blood counts, liver enzymes, renal functions, total cholesterol, blood sugar, and thyroid hormones, at the first visit were acquired. The presence of other etiologies for liver injuries were intensively sought, and the results of imaging studies of the liver, ultrasonography (US) or computed tomography (CT) were also investigated.

\section{Types of disease}

The eating disorder is categorized as $\mathrm{AN}$ or $\mathrm{BN}$, according to the criteria in the DSM-4. AN is characterized as anorexia and remarkable weight loss, while patients of $\mathrm{BN}$ are defined as those with repeated self vomiting after overeating and keeping normal range of weight.

\section{Statistics}

Statistical analysis in the current study was performed using SPSS 14.0J for Windows (SPSS Japan Inc., Tokyo, Japan). Chi-square analysis and Fisher's exact test were used for comparison of clinical parameters between patients with and without liver injuries. Correlation analysis and logistic regression analysis were performed to elucidate correlation among clinical parameters and to determine which variables significantly contributed to the liver injuries, respectively. We regarded the difference as significant if the calculated $\mathrm{p}$ value was less than 0.05 .

\section{Results}

The clinical characteristics of 25 patients enrolled in this study are shown in Table 1 . Twelve patients among the enrolled 25 patients were referred to our hospital from other clinics or hospitals, and no nutritional treatment had been previously performed for these patients. All patients were female. The mean age of all patients was $27.0 \pm 9.0$ years old (mean $\pm \mathrm{SD}$ ). Mean body weight and body mass index (BMI) was $37.3 \mathrm{~kg}$ and $15.2 \mathrm{~kg} / \mathrm{m}^{2}$, respectively. Abnormality in liver enzyme level was observed in 13 out of 25 patients $(52 \%)$. Elevation of serum alanine aminotransferase of greater than $37 \mathrm{IU} / \mathrm{L}$, was found in 13 out of 25 (52\%), while mild elevated alkaline phosphatase was noted in 1 patient, indicating that hepatocellular injuries were dominant in the liver injuries observed in patients with an eating disorder. No patients demonstrated elevation of serum total bilirubin or marked jaundice.

In Table 1, we demonstrated a comparison between patients with an eating disorder in whom elevated alanine aminotransferase (ALT) was present (group A, n=13) and absent (group $\mathrm{B}, \mathrm{n}=12$ ). The median of serum ALT values in each group was 92 [39-438] IU/L and 18 [4-30], respectively. Previous medical treatments were performed in 7 patients in group A and in 5 patients in group B, and we confirmed that there was no causal relationship between liver injury and medications given to the patients in group A. As for subtype of the eating disorder, all patients in group A were $\mathrm{AN}$, while 9 and 3 patients were $\mathrm{AN}$ and $\mathrm{BN}$ in group $\mathrm{B}$, respectively. The mean age was 24.5 in group A and 29.8 in group B, and thus patients with elevated ALT tended to be younger than those without elevated ALT, yet there was no statistically significant difference $(\mathrm{p}=0.139)$. The period between the onset of the disorder and visit to our hospital was 4.2 years in group A and 8.9 years in group B, and patients with elevated ALT had a shorter duration since the onset of eating disorders $(\mathrm{p}=0.037)$. BMI of the patients was significantly lower in patients in group A than those in group B (13.5 vs. 17.3, p=0.011). We did not detect a significant difference in thyroid hormones, thyroid stimulating hormone, total cholesterol, triglycerides, or fasting blood glucose. We performed correlation analysis to determine any correlation among clinical parameters listed in Table 1, yet 
Table 1. Comparison of Clinical Parameters between Patients with the Presence (Group A) and Absence (group B) of Elevated ALT

\begin{tabular}{|c|c|c|c|}
\hline & Group A $(n=13) * *$ & Group B $(n=12) * *$ & $\mathrm{p}$ value $* * *$ \\
\hline Serum ALT $(\mathrm{IU} / \mathrm{L}) *$ & $92[39-438]$ & $18[4-30]$ & N/A \\
\hline Sex (male: female) & $0: 13$ & $0: 12$ & $>0.999$ \\
\hline Type (AN/BN) & $13 / 0$ & $9 / 3$ & 0.096 \\
\hline Age (years old) & $24.5 \pm 8.2$ & $29.8 \pm 9.3$ & 0.139 \\
\hline $\begin{array}{l}\text { The period since onset of the } \\
\text { disorder (year) }\end{array}$ & $4.2 \pm 5.2$ & $8.9 \pm 5.4$ & 0.037 \\
\hline Body Mass Index $\left(\mathrm{kg} / \mathrm{m}^{2}\right)$ & $13.5 \pm 1.6$ & $17.3 \pm 4.6$ & 0.011 \\
\hline FreeT3 (ng/dL) * & $1.7 \pm 0.5$ & $2.2 \pm 0.7$ & 0.088 \\
\hline FreeT4 $(\mathrm{ng} / \mathrm{dL}) *$ & $1.11 \pm 0.20$ & $1.06 \pm 0.18$ & 0.460 \\
\hline $\mathrm{TSH}(\mu \mathrm{g} / \mathrm{dL}) *$ & $2.658 \pm 2.903$ & $2.481 \pm 1.778$ & 0.862 \\
\hline Total cholesterol $(\mathrm{mg} / \mathrm{dL}) *$ & $198 \pm 67$ & $232 \pm 89$ & 0.312 \\
\hline Triglycerides $(\mathrm{mg} / \mathrm{dL}) *$ & $134 \pm 214$ & $89 \pm 86$ & 0.690 \\
\hline Fasting blood sugar $(\mathrm{g} / \mathrm{dL}) *$ & $79 \pm 9$ & $89 \pm 11$ & 0.027 \\
\hline Hemoglobin $(\mathrm{g} / \mathrm{L})$ & $12.0 \pm 1.0$ & $12.0 \pm 1.4$ & 0.829 \\
\hline
\end{tabular}

* The reference values in our hospital: ALT 3-38 IU/L, Free T3 2.3-4.3 ng/dL, Free T4 1.00-

$1.64 \mathrm{ng} / \mathrm{dL}$, TSH $0.450-4.950 \mu \mathrm{g} / \mathrm{dL}$, Total cholesterol 130-250 mg/dL, Triglycerides 30-180

$\mathrm{mg} / \mathrm{dl}$, Fasting blood sugar 80-100 g/dL, Hemoglobin 13.0-16.9 g/dL.

** The continuous values are expressed as mean \pm standard deviation.

*** p value was calculated using chi-square test and Fisher's exact test.

there was no significant correlation except for that between age and duration $(\mathrm{r}=0.609, \mathrm{p}=0.001)$. Logistic regression analysis using age, BMI, the duration since onset, Free T4 and fasting blood sugar as variables revealed that only BMI significantly contributed to the development of liver injuries $(\mathrm{OR}=3.46 ; 95 \%$ CI 1.06-11.34, $\mathrm{p}=0.041)$. US and CT was performed in 7 and 1 patients in group A, respectively, and none of them demonstrated any abnormalities, including fatty liver.

\section{Discussion}

Mild to moderate elevations of aminotransferases are occasionally observed in patients with AN. A communitybased study suggested that $12.2 \%$ of patients with AN showed an elevation of aminotransferase (3). In Japan, the frequency of hepatocellular damage on admission or prior to the effective therapy was reported to be $20 \%$ (2) or $29 \%$ (11). In the current study, we enrolled patients with eating disorders who had no previous nutritional treatment to exclude the possible hepatotoxic effect of nutritional support, and an elevation of ALT was observed in 52\% (13/25), and all 13 patients were categorized as AN. Although the number of patients enrolled in this study was relatively small, the prevalence of hepatocellular injuries in AN was higher than in the previous reports, both of which were performed in 1990s.

It is noteworthy that the BMI was lower (13.5 vs. 17.3, $\mathrm{p}=0.011$ ) and the duration since the onset of AN was shorter
(4.2 years vs. 8.9 years, $p=0.037$ ) in patients with elevated ALT, compared to those with normal ALT. The age was younger in patients with elevated ALT, even though the difference was not significant ( 24.5 vs. 29.8 , p=0.139). Recently Tajiri et al reported a similar finding that AN patients with higher ALT had a shorter duration of the disease and lower BMI (9). Basal metabolic rate (BMR) in females is the highest in the age group of 12-14 years old, 1,359 kcal/ day, and gradually declines as the age increases (14). Thus, it is reasonable that younger AN patients may suffer more metabolic damage from nutritional deficiency. Moreover, we noted that 9 patients with normal ALT had a history of a relatively mild eating disorder, while 13 patients with liver injuries developed a more severe eating disorder with lower BMI, which probably contributed to earlier consult or referral to our hospital and consequently resulted in the shorter duration of the disease. In addition, multivariate analysis revealed that only BMI was a significant determinant for liver injuries. Taken together, our findings suggest that the severity of AN, characterized by lower BMI, might play an important role in the development of hepatocellular damage.

It still remains unclear why patients with AN develop hepatocellular injury. Fatty liver, or excess accumulation of fat in hepatocytes, has been demonstrated in case series by imaging studies or histological studies $(7,10,15)$. By contrast, another histological study of an AN patient with severe liver damage failed to demonstrate any fat infiltration (16). A recent study showed that detection of fatty liver by US was limited to a small portion of AN patients with elevated ALT 
$(5 / 18 ; 28 \%)$ (9). Taken together, the excess accumulation of fat in the liver definitely plays a role for the induction of hepatocellular injuries in patients with $\mathrm{AN}$, yet should not be considered as a single cause. Indeed, our US/CT studies failed to demonstrate fatty liver in all 8 patients tested in the current study. Clearly, hepatocellular injuries in patients with $\mathrm{AN}$ are multifactorial, and it is reasonable to assume that several factors triggered by general malnutrition contribute to the development of hepatocellular damage. Other etiologies explaining hepatocellular injuries have included acute hypoperfusion of the liver (6), oxidative stress and iron deposition (10), and microcirculatory failure or hypoxia (16). Obviously histological examination of the liver would greatly help to clarify how liver injuries were caused in patients with AN, yet we were very reluctant to perform invasive procedures such as liver biopsy in patients with AN.

In conclusion, we demonstrated in this study that AN patients with elevated ALT prior to any nutritional treatment were characterized by lower BMI and shorter duration from the onset of the disease, and a lower BMI might significantly contribute to hepatocellular injuries. Liver injuries in patients with an eating disorder could result in severe hepatic failure or a fatal outcome (4-7), and thus should not be overlooked. Accumulation of AN cases with liver injuries is clearly necessary for solving the mechanisms by which malnutrition in AN patients causes hepatocellular damage.

\section{References}

1. American Psychiatric Association. Diagnostic and Statistical Manual of Mental Disorders. 4th ed. Washington DC, 1994.

2. Mine T, Ogata E, Kumano H, Kuboki T, Suematsu H. An analysis of liver injury in patients with AN. Research reports from investigational group for anorexia nervosa. Ministry of Health and Welfare, 1992 (in Japanese).

3. Miller K, Grinspoon S, Ciampa J, Hier J, Herzog D, Klibanski A. Medical findings in outpatients with anorexia nervosa. Arch Intern Med 165: 561-566, 2005.

4. Furuta S, Ozawa Y, Maejima K, et al. Anorexia nervosa with severe liver dysfunction and subsequent critical complications. Intern Med 38: 575-579, 1999.

5. Di Pascoli L, Lion A, Milazzo D, Caregaro L. Acute liver damage in anorexia nervosa. Int J Eat Disord 36: 114-117, 2004.

6. De Caprio C, Alfano A, Senatore I, Zarrella L, Pasanisi F, Contaldo F. Severe acute liver damage in anorexia nervosa: two case reports. Nutrition 22: 572-575, 2006.

7. Sakada M, Tanaka A, Ohta D, et al. Severe steatosis resulted from anorexia nervosa leading to fatal hepatic failure. J Gastroenterol 41: 714-715, 2006.

8. Terada M, Kidani H, Masuda S, Nakanuma Y. A case of nonalcoholic steatohepatitis due to anorexia nervosa revealed hepatic failure with marked hepatomegaly. Kanzo 36: 617-621, 1997 (in Japanese).
9. Tajiri K, Shimizu Y, Nakayama Y, et al. A clinical analysis of liver injury in patients with AN. Kanzo 44 (suppl 3): A548, 2003 (in Japanese).

10. Tajiri K, Shimizu Y, Tsuneyama K, Sugiyama T. A case report of oxidative stress in a patient with anorexia nervosa. Int J Eat Disord 39: 616-618, 2006.

11. Ozawa Y, Shimizu T, Shishiba Y. Elevation of serum aminotransferase as a sign of multiorgan-disorders in severely emaciated anorexia nervosa. Intern Med 37: 32-39, 1998.

12. Quigley E, Marsh M, Shaffer J, Markin R. Hepatobiliary complications of total parenteral nutrition. Gastroenterology 104: 286301, 1993.

13. Paquot N, Delwaide J. Fatty liver in the intensive care unit. Curr Opin Clin Nutr Metab Care 8: 183-187, 2005.

14. Ministry of Health, Labor and Welfare. Dietary reference intakes for Japanese. Dai-ichi Shuppan, 2005 (in Japanese).

15. Yaryura-Tobias J, Pinto A, Neziroglu F. Anorexia nervosa, diabetes mellitus, brain atrophy, and fatty liver. Int $\mathrm{J}$ Eat Disord 30: 350-353, 2001.

16. Yanase $K$, Takakawa $T$, Kawaguchi $T$, et al. A case of anorexia nervosa with liver injury. Shoukaki-shinshin-igaku 12: 25-28, 2005 (in Japanese).

(C) 2008 The Japanese Society of Internal Medicine http://www.naika.or.jp/imindex.html 